\title{
Functional disparities among BCL-2 members in tonsillar and leukemic B-cell subsets assessed by BH3-mimetic profiling
}

\author{
Victor Peperzak ${ }^{1}$, Erik Slinger ${ }^{1,2}$, Johanna Ter Burg ${ }^{1,2}$ and Eric Eldering ${ }^{\star, 1,3}$
}

For successful treatment of malignant B-cells it is crucial to understand intrinsic survival requirements in relation to their normal progenitors. Long-lived humoral immunity as well as most B-cell malignancies, originate in the germinal center (GC). Murine GC B-cells depend on pro-survival protein MCL-1, but not BCL-XL. In contrast, naive and memory B-cells depend on BCL-2, but not BCL-XL or MCL-1. For human B-cell subsets, the functional relationships among BCL-2 members are unclear, and also if and how they shift after malignant transformation. We here dissect these aspects in human tonsil and primary leukemia (CLL) cells by single and combined treatment with novel, highly specific BH3-mimetics. We found that MCL-1 expression in GC B-cells is regulated posttranslationally and its importance is highlighted by preferential binding to pro-apoptotic BIM. In contrast, BCL-XL is transcriptionally induced and binds solely to weak sensitizer BIK, potentially explaining why BCL-XL is not required for GC B-cell survival. Using novel BH3-mimetics, we found that naive and memory B-cells depend on BCL-2, GC cells predominantly on MCL-1, whereas plasma cells need both BCL-XL and MCL-1 for survival. CLL cells switch from highly sensitive for BCL-2 inhibition to resistant after CD40-stimulation. However, combined inhibition of BCL-2, plus BCL-XL or MCL-1 effectively kills these cells, thus exposing a weakness that may be therapeutically useful. These general principles offer important clues for designing treatment strategies for B-cell malignancies.

Cell Death and Differentiation (2017) 24, 111-119; doi:10.1038/cdd.2016.105; published online 30 September 2016

The intrinsic apoptotic pathway is controlled by the BCL-2 protein family. Expression of the pro-survival members, namely BCL-2, BCL-XL, BCL-W, MCL-1, BFL-1 and BCL-B, varies greatly and strongly depends on the cell type, its environment and activation state. ${ }^{1}$ Understanding the regulation and degree of expression is key to determine which pro-survival protein(s) is (are) essential for survival of certain cell types at different stages of differentiation or activation.

An important distinction can be made for the BH3-only proteins of the BCL-2 family. Although certain members can induce apoptosis by directly binding to effectors BAX and BAK (BIM, BID and P53 up-regulated modulator of apoptosis (PUMA); also referred to as activators), other members can only indirectly regulate apoptosis by sequestering pro-survival proteins (BAD, NOXA, BIK and so on; referred to as sensitizers). ${ }^{1}$

Overexpression of pro-survival BCL-2 family members can allow survival of proliferating cells that would otherwise be deleted via apoptosis. As a consequence, oncogenic mutations that can arise in the germinal center (GC) combined with overexpression of pro-survival BCL-2 proteins, facilitates cancer development. ${ }^{1,2}$ BH3-mimetics were developed to block specific pro-survival BCL-2 proteins and force cells that depend on them to undergo apoptosis. BCL-2-specific BH3mimetic ABT-199 (Venetoclax) has shown great promise in the treatment of chronic lymphocytic leukemia (CLL), as CLL cells uniformly over-express BCL-2. ${ }^{3}$ Like BCL-2, MCL-1 is often over-expressed in different B-cell malignancies, such as

\footnotetext{
${ }^{1}$ Department of Experimental Immunology, Academic Medical Center, University of Amsterdam, Amsterdam, The Netherlands; ${ }^{2}$ Department of Hematology, Academic Medical Center, University of Amsterdam, Amsterdam, The Netherlands and ${ }^{3}$ Department of Lymphoma, Myeloma Center Amsterdam, LYMMCARE, Amsterdam, The Netherlands

${ }^{*}$ Corresponding author: E Eldering, Department of Experimental Immunology, Academic Medical Center, University of Amsterdam, Meibergdreef 9 , Amsterdam 1105 AZ, The Netherlands. Tel: +31 20566 7018; Fax: +31 20566 9756; E-mail: e.eldering@amc.uva.nl

Abbreviations: BAD, Bcl-2 associated agonist of cell death; BAK, Bcl-2 antagonist/killer; BAX, Bcl-2-associated X; BBC3, Bcl-2-binding component 3; BCL-2, B-cell lymphoma 2; BCL2L11, B-cell lymphoma 2 like 11; BCL-B, B-cell lymphoma B; BCL-W, B-cell lymphoma W; BCL-XL, B-cell lymphoma extra-large; BCR, B-cell receptor; $\mathrm{BFL}-1, \mathrm{BCl}-2$-related gene in fetal liver 1; BH3, Bcl-2 homology domain 3; BID, Bh3-interacting domain death agonist; BIK, Bcl-2 interacting killer; BIM, Bcl-2 interacting mediator of cell death; BIMEL, Bcl-2 interacting mediator of cell death extra-long isoform; BIML, Bcl-2 interacting mediator of cell death long isoform; $\mathrm{BIMS}$, Bcl-2 interacting mediator of cell death short isoform; BMF, Bcl-2 modifying factor; Bn, Naive B cells; BTRC, Beta-transducin repeat containing; $\beta$ TrCP1, Beta-transducin repeat containing E3 ubiquitin protein ligase pseudogene 1; CB, Centroblasts; CC, Centrocytes; CHX, Cycloheximide; CLL, Chronic lymphocytic leukemia; DZ, Dark zone; FACS, Fluorescence-activated cell sorting; FBW7 and FBXW7, F-Box and WD repeat domain-containing 7; FCRFludarabine, Cyclophosphamide and Rituximab; FL, Follicular lymphoma; GC, Germinal center; Hprt, Hypoxanthine-guanine phosophoribosyltransferase; HUWE1, HECT UBA and WWE domain-containing 1; IP, Immunoprecipitation; LZ, Light zone; MCL-1, Myeloid cell leukemia 1; Mem, memory B cells; MLPA, Multiplex ligation-dependent probe amplification; Mule, Mcl-1 ubiquitin ligase E3; NOXA, Latin for 'damage'; OA, Okadaic acid; PBS, Phosphate-buffered saline; PC, Plasma cells; PCR, Polymerase chain reaction; PP2A, Protein phosphatase 2; PUMA, P53 upregulated modulator of apoptosis; RNA, Ribonucleic acid; RT-MLPA, Reverse transcriptase multiplex ligation-dependent probe amplification; TGF- $\beta$, Transforming growth factor beta
}

Received 11.4.16; revised 25.8.16; accepted 31.8.16; Edited by A Strasser; published online 30.9.16 
diffuse large B-cell lymphoma, follicular lymphoma (FL), CLL and multiple myeloma. ${ }^{4-6}$ In addition to BCL-2-specific $\mathrm{BH}$ mimetics, novel BH3-mimetics have become available for in vitro use that specifically target MCL-1 (A-1210477) or BCLXL (WEHI-539). ${ }^{7,8}$

Most lymphomas derive from GC B cells or their descendants. ${ }^{9}$ Thus, predicting efficacy of $\mathrm{BH} 3-$ mimetics in $\mathrm{B}$-cell malignancies requires detailed insight into expression of BCL-2 family proteins, their interaction profile and sensitivity to BH3-mimetics in healthy B cells. High-level MCL-1, $B C L-X L$ and reduced $B C L-2$ protein expression has been previously detected in the human and murine $\mathrm{GC}^{10-13}$ In addition, transcriptional induction of BFL-1 was observed by gene expression profiling in the human and murine GC light zone (LZ). ${ }^{14}$ Although MCL-1 and BCL-XL proteins are both highly expressed in murine GC B cells, only MCL-1 appeared to be important for their survival. ${ }^{13}$ The divergent roles of MCL-1 and BCL-XL in GC B cells still remain unexplained, and it is unknown if this also holds for human $B$ cells.

The aim of our current study is twofold; first, we aim to investigate the expression, regulation and dependence on pro-survival BCL-2 family members in healthy primary human $B$ cells from the tonsil, including GC B cells (discriminating centroblasts (CB) from the GC dark zone (DZ) and centrocytes (CC) from the LZ), and plasma cells (PC). Second, BH3profiling with peptides has been used to predict dependence on pro-survival BCL-2 family members. ${ }^{15}$ Here, we use another approach using $\mathrm{BH} 3-$ mimetic compounds that have become available and selectively inhibit either BCL-2, BCL-XL or MCL-1. Recently, an innovative method, called mitopriming, has tested such novel BH3-mimetics and confirmed their selectivity and potency. ${ }^{16}$ To exploit potential differences in sensitivity between healthy and malignant B cells we also used primary CLL cells. These cells normally respond well to inhibition with $\mathrm{ABT}-199,{ }^{3}$ but upregulate BCL-XL, MCL-1 and BFL-1 on stimulation via CD40, mimicking the protective lymph node microenvironment and making them resistant to ABT-199 and other conventional CLL drugs. ${ }^{17}$ CLL cells can therefore be used as a model to study the dependence of primary malignant $B$ cells on expression of the different BCL-2 family members.

\section{Results}

BCL-2 family members are highly differentially expressed in human B-cell subsets. To examine gene expression of the different BCL-2 family members, B-cell subsets were isolated from human tonsil specimens and subjected to multiplex ligation-dependent probe amplification assay (MLPA) covering both pro-apoptotic and pro-survival members of the BCL-2 family (Figures 1a-c; Supplementary Figure 1). Tonsil B cells were separated into naive $B$ cells, memory B cells, PC or GC B cells based on expression of IgD and CD38 using flow cytometry. GC B cells were further subdivided into $\mathrm{CB}$ or $\mathrm{CC}$ based on expression of chemokine receptor CXCR4 and cell size (Figure 1a). We confirmed mRNA expression of pro-survival members using more sensitive quantitative real time polymerase chain reaction
(qPCR) (Figure 1d), which also allowed quantification of BCL-2 family expression in the small PC population isolated from the tonsil. Combined, this revealed that BCL2 gene expression was virtually shut down when $B$ cells enter the $\mathrm{GC}$ reaction. In contrast, gene expression of $B C L X$ and $B F L 1$ was induced in the $L Z$ of the GC, whereas gene expression of $M C L 1$ was significantly increased in PC only (Figures $1 \mathrm{~b}-\mathrm{d}$ ). Besides MCL1, a significant increase in BCLX gene expression was observed in PC (Figure 1d). The expression pattern in memory $B$ cells was not significantly different than in naive $B$ cells (Figures $1 b-d)$ ). In addition to the differential regulation of pro-survival BCL-2 family members, we also observed significantly increased gene expression of pro-apoptotic members BIM, BID, PUMA and especially BIK when B cells enter the GC reaction (Figure 1; Supplementary Figure 2). Increased gene expression of these pro-apoptotic BCL-2 family members was also observed in PC (Supplementary Figure 3).

Pro-survival BCL-2 proteins are transcriptionally regulated in tonsillar B cells, except MCL-1, that is regulated at the post-transcriptional level. Protein expression of differentially expressed BCL-2 family members was monitored by western blot analysis on cells sorted as in Figure 1a. In general, gene expression was largely consistent with expression at the protein level (Figures $2 \mathrm{a}$ and b). Unlike $B C L X$ and BFL1, increased MCL1 gene expression was not observed when $B$ cells enter the GC reaction (Figures $1 \mathrm{a}-\mathrm{d}$ ). MCL-1 protein however is highly induced in GC B cells as measured by western blot and fluorescence microscopy (Figures 2a-c). Quantification of multiple experiments revealed that increased expression of $M C L-1$ and $B C L-X L$, or decreased expression of BCL-2, in GC B cells did not differ between $\mathrm{CB}$ or $\mathrm{CC}$ from the $\mathrm{DZ}$ or $\mathrm{LZ}$, respectively (Figure $2 b$ ). Although induction of BFL-1 protein expression in $\mathrm{CC}$ seems to follow its regulation at the transcriptional level (Figures $1 \mathrm{~b}-\mathrm{d}$ and $2 \mathrm{a}$ ), the currently available tools to detect BFL-1 protein did not allow quantification relative to naive $B$ cells. In contrast to $\mathrm{GC} B$ cells, high-level MCL-1 protein expression was not observed in the $\mathrm{T}$ cell zone surrounding the tonsillar GC as observed by fluorescence microscopy (Figure 2c). Comparing MCL-1 protein expression to gene transcription suggested that MCL-1 protein stability is increased specifically in GC B cells (Figure 3a). Measuring MCL-1 protein turnover using translation inhibitor cycloheximide in highly purified naive $B$ cells versus $\mathrm{GC} B$ cells indeed revealed that MCL-1 protein stability in GC B cells is strongly increased (Figure 3b; Supplementary Figures $4 a$ and b). MCL-1 protein half-life is dictated by the combined action of a selection of specific kinases, phosphatases, deubiquitinases and ubiquitin ligases. ${ }^{18}$ Which of these players is responsible for the regulation of $\mathrm{MCL}-1$ stability in GC B cells is currently unknown, although we did observe a significant decrease of known MCL-1-specific ubiquitin ligases in GC B cells (Figure $3 \mathrm{c}$ ). ${ }^{18}$ In addition, we observed that inhibition of the protein phosphatase 2 (PP2A) complex using okadaic acid $(O A)$ reduced $M C L-1$ stability in GC B cells (Figure $3 d$ ). The latter findings are in line with the observation that protein phosphatases, such as PP2A, are highly active in GC B cells. ${ }^{19}$ 
a

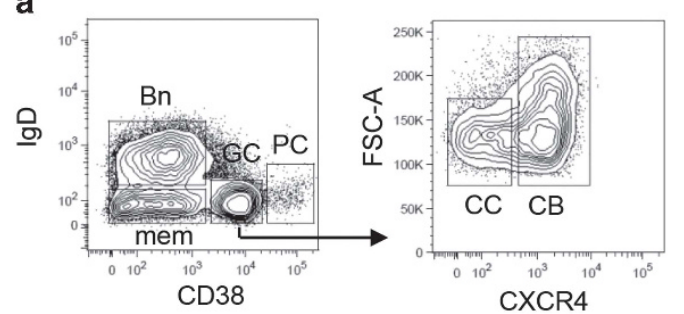

b
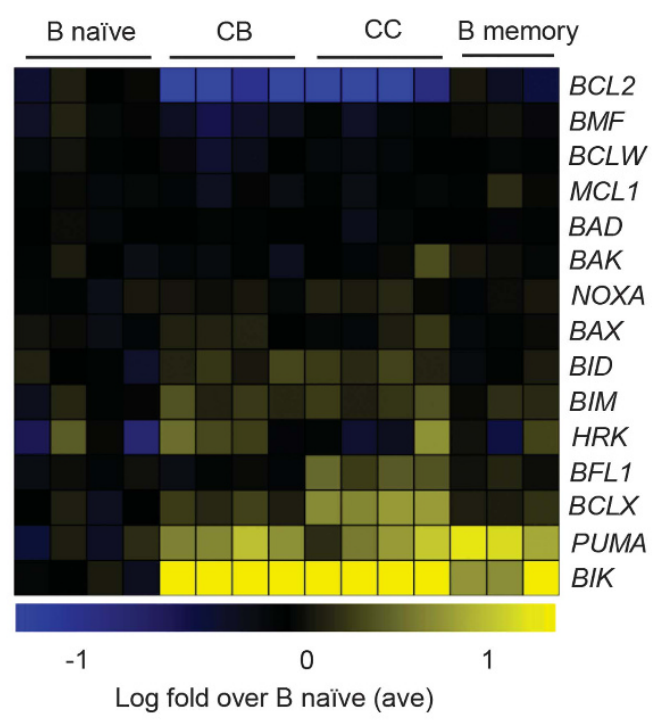

c

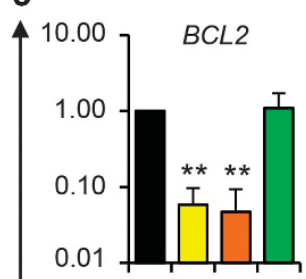

$100.0] \quad$ MCL1

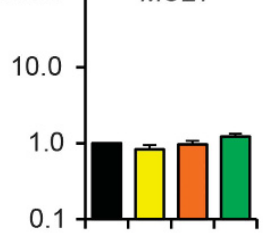

$100.0] \begin{aligned} & \text { BCL2L1 } \\ & \text { (BCLXL) }\end{aligned}$

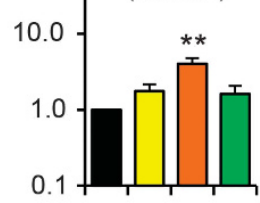

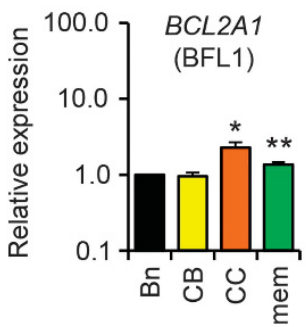

d
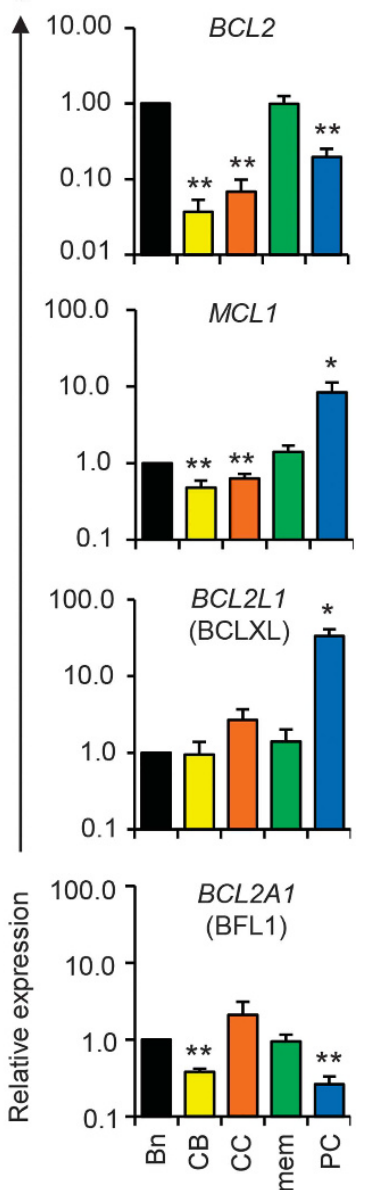

Figure 1 Differential gene expression of $B C L-2$ family members in primary human $B$ cells. (a) Gating strategy for isolation of naive $B$ cells $\left(B n, \lg D^{+} C D 38^{-}\right)$, memory $B$ cells (mem, IgD- $\left.\mathrm{CD} 38^{-}\right)$, plasma cells $\left(\mathrm{PC}, \lg \mathrm{D}^{-} \mathrm{CD} 38^{++}\right)$and germinal center $\mathrm{B}$ cells $\left(\mathrm{GC}, \operatorname{lgD}{ }^{-} \mathrm{CD} 38^{+}\right)$from the human tonsil after gating on $\mathrm{CD} 19^{+} \mathrm{CD} 3^{-} \mathrm{B}$ cells. $\mathrm{GC} B$ cells were further subdivided in either centroblast (CB, CXCR4 ${ }^{+} F S C-A^{+/}$) or centrocyte (CC, CXCR4 ${ }^{-}$FSC- $A^{-}$) cells (right panel). (b) Expression profiling by RT-MLPA of human B cells purified directly ex vivo as shown in a. Gene induction of pro- and anti-apoptotic molecules is represented in a heat-map after log2 transformation of expression levels, relative to averaged values of naive $B$ cells. Data is shown from four individual patients (three for memory B cells). (c) Averages of values shown in (b) relative to naive B cells. (d) Real time qPCR of indicated BCL-2 family members in purified cells as shown in a, corrected for expression of household gene Hprt and relative to naive $B$ cells. Data are average of three $(B C L X, B F L 1)$ or five $(B C L 2, M C L 1)$ experiments with s.e.m. Statistics were calculated in relation to $\mathrm{Bn}$ cells. ${ }^{*} P \leqslant 0.05,{ }^{*} P \leqslant 0.01$

Selective binding patterns of BCL-XL and MCL-1 in tonsillar B cells. To further probe the differential roles of MCL-1 and BCL-XL in tonsil B cells, we performed immunoprecipitation (IP) experiments to detect their binding partners. These IP experiments were performed on total $B$ cells purified from the tonsil and this population consists mainly of GC B cells (CD38 $\left.{ }^{+} ; 36 \%\right)$, memory B cells (CD38- $\left.{ }^{-} \mathrm{IgD}^{-} ; 34 \%\right)$ and naive $B$ cells $\left(C D 38^{-} \operatorname{lgD}^{+} ; 29 \%\right)$, with only a small fraction of PC (CD38 $\left.{ }^{++} ; 1.3 \%\right)$ (Supplementary Figure $\left.5 \mathrm{a}\right)$. As protein expression of BCL-2, BCL-XL and MCL-1 in tonsil B cells is strictly cell-type specific (Figure $2 \mathrm{~b}$ ), with almost $90 \%$ of BCL-2 protein expressed in naive and memory $B$ cells and over $80 \%$ of $\mathrm{MCL}-1$ or $\mathrm{BCL}-\mathrm{XL}$ protein expressed in $\mathrm{GC} \mathrm{B}$ cells (Supplementary Figure 5), we deduced that IPs for MCL-1 or BCL-XL are mostly representative for GC B cells. These IP experiments revealed that MCL-1 was predominantly bound to BIM (Figure 4a) and not to other proapoptotic proteins such as BIK, PUMA or BID (Figure 4a). Expression of NOXA was barely detectable, but long exposures in some western blots did show a specific association with MCL-1, as expected based on the known specificity of NOXA (Supplementary Figure 6a). Aspecific bands in the IP fraction were visualized by incubation with labeled secondary antibodies only, and indicated by stars (Figure $4 a$ ). Extended running of the western blot containing IP fractions revealed that the $B I M_{E L}$ band was partly masked by an aspecific background band, but that in addition to $\mathrm{BIM}_{S}$ and $B I M_{L}$, the $B I M_{E L}$ form is also associated with MCL-1 (Supplementary Figure 6a). BCL-XL, on the other hand, was not associated with BIM, but was associated with BIK instead, and no binding of BCL-XL to PUMA, BID or NOXA was detected (Figure 4a; Supplementary Figure 6a). MCL-1 IP could not completely deplete the total lysate of BIM, which either suggests saturation of the beads, or that part of the BIM protein pool was also associated with other proteins than MCL-1. BCL-2 IPs indeed showed that a small but detectable fraction of BIM was also bound to BCL-2. In addition to BIM, PUMA was found associated to BCL-2 (Supplementary 


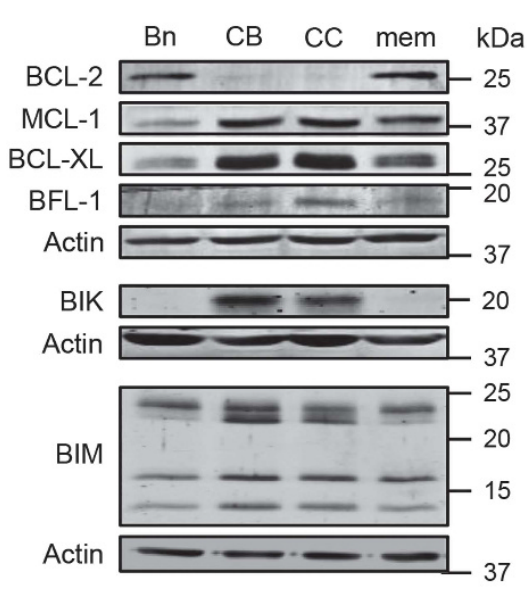

c

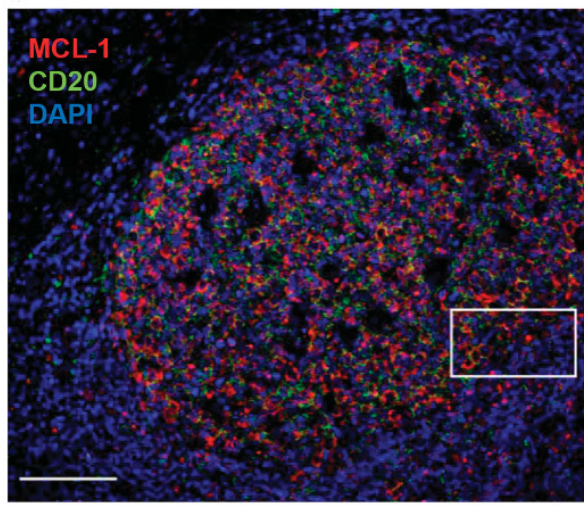

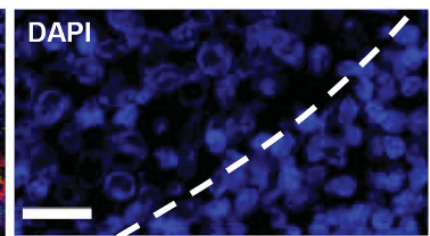
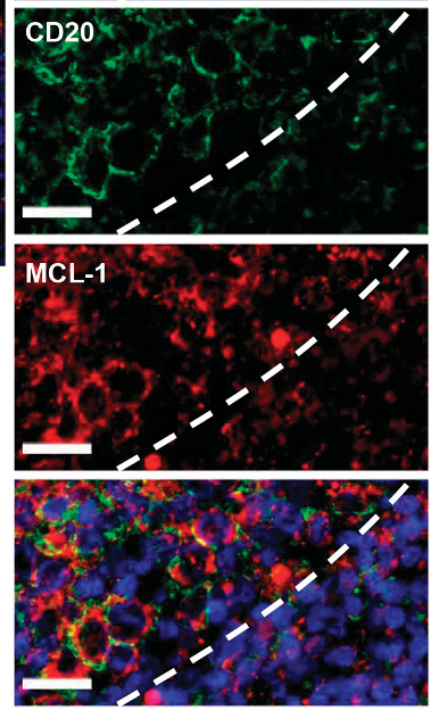

b
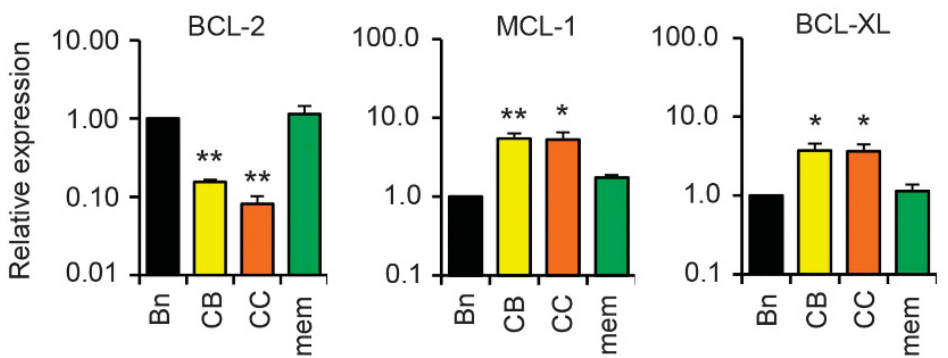

Figure 2 Differential protein expression of BCL-2 family members in primary human B cells. (a) Western blot analysis for indicated BCL-2 family members in tonsil B cells, fluorescence-activated cell sorting (FACS)-purified as shown in Figure 1a. Data is representative of two (BFL-1, BIK and BIM) to four (BCL-2, MCL-1 and BCL-XL) independent experiments. (b) Quantified western blots shown in a corrected for expression of Actin and relative to naive B cells. BFL-1 expression could not be quantified due to the low expression level. Data are average values of three to four different experiments with S.E.M. (c) Fluorescence microscopy image of a tonsil section showing MCL-1 protein staining in a CD20 ${ }^{+} \mathrm{GC}$ in the tonsil. Scale bar, $100 \mu \mathrm{m}$ in overview image (left panel) and $20 \mu \mathrm{m}$ in selection (right panel). Images are representative of three individual experiments. Statistics were calculated in relation to Bn cells. ${ }^{*} P \leqslant 0.05,{ }^{*} P \leqslant 0.01$

Figure $6 \mathrm{~b}$ ), whereas BIK was not bound to BCL-2 (Supplementary Figure $6 \mathrm{c}$ ). As almost $90 \%$ of $\mathrm{BCL}-2$ protein in the total B-cell lysate is derived from naive or memory B cells, these observed associations are also mostly reflective of these cell subsets. Thus, MCL-1 associates with BIM and NOXA and BCL-XL with BIK in GC B cells, whereas BCL-2 associates with PUMA and BIM in naive and/or memory B cells. Unfortunately, with the currently available BFL-1 antibodies, we were unable to successfully perform BFL-1 IPs to test a possible interaction of BIM or BIK to BFL-1. To test if the novel MCL-1-inhibitor A-1210477 could abrogate the interaction between MCL-1 and BIM, IP experiments were performed in the presence of A-1210477 and demonstrated a strong reduction of the interaction between MCL-1 and BIM (Figure 4b). Similarly, BCL-XL-BIK complexes could be dissociated using the BCL-XL-inhibitor WEHI-539 (Figure 4c). Combined, our data reveal a clear distinction in regulation as well as functional interactions between MCL-1 and $\mathrm{BCL}-\mathrm{XL}$ in human tonsil B-cell subsets.

BH3-mimetic profiling reveals a divergent dependence on pro-survival BCL-2 family members in healthy B cells and exposes weaknesses in resistant leukemic $B$ cells. In mouse models, expression of MCL-1 is essential for GC B-cell survival. ${ }^{13}$ The importance of MCL-1 expression for human B cells is currently unknown and was tested with A-1210477 by treating tonsil cells with different doses of the inhibitor, followed by flow cytometric determination of cell viability after gating on the different $B$ cells subsets, as shown in Figure 1a. This revealed increased sensitivity of GC B cells and $\mathrm{PC}$ to inhibition of MCL-1 as compared with either naive or memory B cells (Figure 5a). A-1210477 is highly specific for MCL-1, although relatively high concentrations are necessary to displace BIM from MCL-1 and promote apoptosis. ${ }^{7}$ It can be expected that more potent inhibitors will reveal bigger differences in MCL-1 sensitivity between cell types in the future. Next, sensitivity to the BCL-XL-specific inhibitor WEHI-539 was tested in a similar manner. Although $\mathrm{BCL}-\mathrm{XL}$ protein expression is strongly increased in GC B cells (Figures $2 a$ and $b$ ), these cells are not sensitive to inhibition with WEHI-539 (Figure 5a). Importantly, this confirms earlier findings in mice in vivo, ${ }^{13}$ and is in line with $\mathrm{BCL}-\mathrm{XL}$ binding to weak $\mathrm{BH} 3$ member $\mathrm{BIK}$. $\mathrm{PC}$ in human tonsil are however sensitive to inhibition of BCL-XL (Figure $5 \mathrm{a}$ ), in correlation with very high $\mathrm{BCL}-\mathrm{XL}$ mRNA expression in PC (Figure 1d), and increased overall expression of BH3 members BIM, PUMA, BIK and BAD (Supplementary Figure 3). Finally, we tested dependence on BCL-2 exclusively using the BCL-2-specific inhibitor ABT-199. Comparable to mouse experiments using ABT-737, that 
blocks both $\mathrm{BCL}-2$ and $\mathrm{BCL}-\mathrm{XL},{ }^{20}$ naive and memory human $B$ cells were highly sensitive to specific BCL-2 inhibition, whereas GC B cells and PC were not (Figure 5a). The differential sensitivities of tonsil subsets for the BH3-mimetics ranged from 10-fold (A-1210477) to >100-fold (WEHI-539, ABT-199).

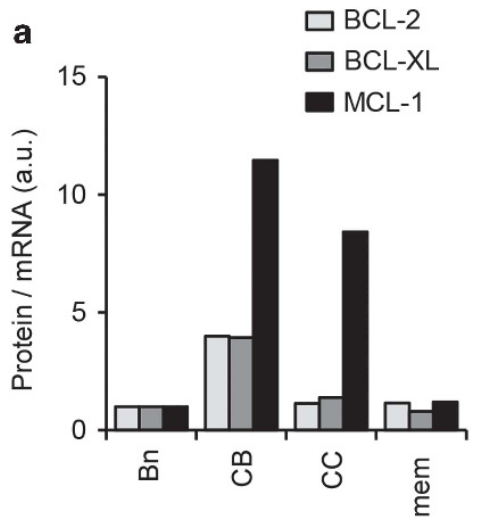

b
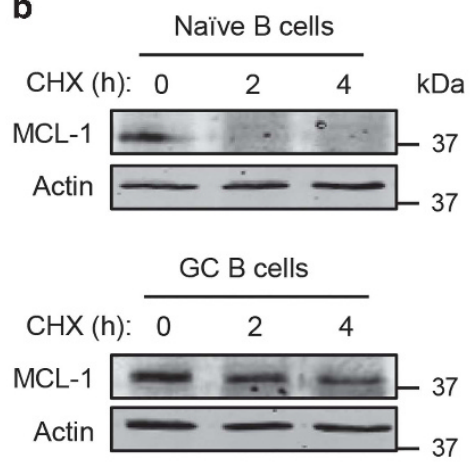

d
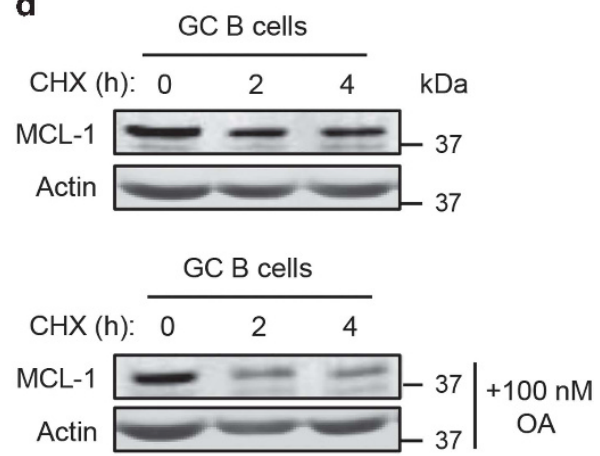
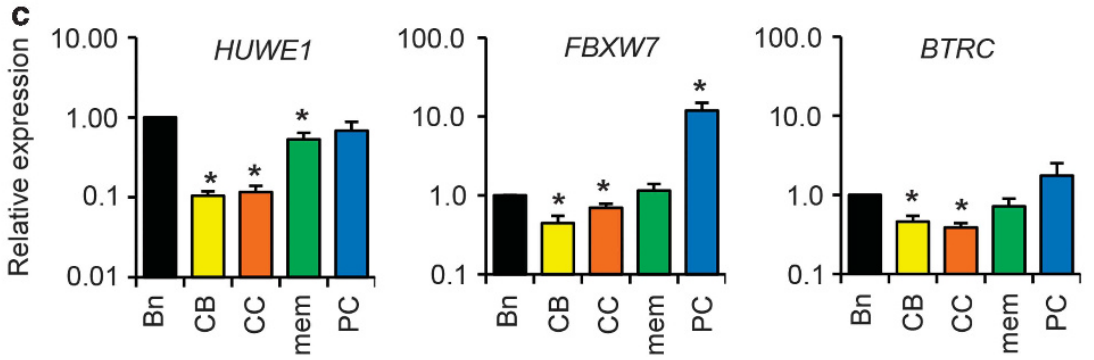

Figure $3 \mathrm{MCL}-1$ protein is stabilized in GC B cells. (a) Ratios of protein (average of quantified western blot experiments shown in Figure 2b, with $n=2-4$ ) over mRNA (average of real time qPCR experiments shown in Figure 1d, with $n=3-5$ ) in B-cell subsets for MCL-1, BCL-2 and BCL-XL expressed in arbitrary units (a.u.) (b) Western blot analysis on FACS-purified naive or GC B cells for MCL-1 and Actin after culture for 0,2 or $4 \mathrm{~h}(\mathrm{~h})$ with cycloheximide (CHX), an inhibitor of protein synthesis. Data are representative of two individual experiments. (c) Real time qPCR of known MCL-1-specific ubiquitin ligases Mule (HUWE1), FBW7 (FBXW7) and $\beta$ TrCP1 (BTRC) in purified cells as shown in Figure 1a, corrected for expression of household gene Hprtand relative to naive B cells. Data are average of five experiments with S.E.M. Statistics were calculated in relation to Bn cells. (d) Western blot analysis on MACS-enriched GC B cells for MCL-1 and Actin after culture for 0,2 or $4 \mathrm{~h}$ (h) with cycloheximide (CHX), with or without PP2A-inhibitor okadaic acid (OA). Cells in (b) and (d) were cultured in standard tissue culture medium (Iscove's modified Dulbecco's media) supplemented with 10\% (v/v) heat-inactivated fetal calf serum and antibiotics, but without additional cytokines. ${ }^{*} P \leqslant 0.05,{ }^{* *} P \leqslant 0.01$

a

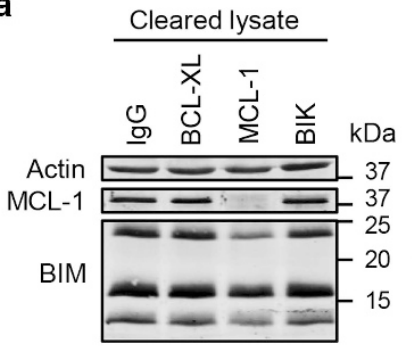

Actin 37

BCL-XL $=-25$

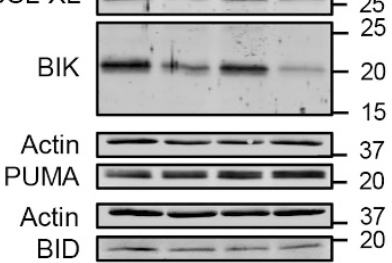

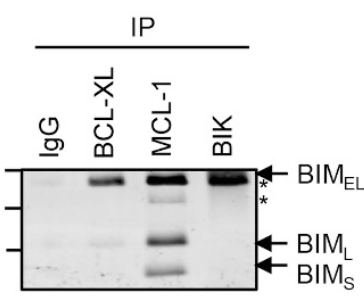

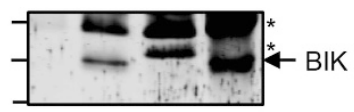

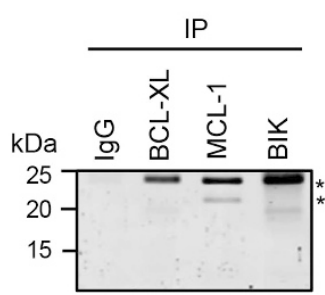

Secondary

only

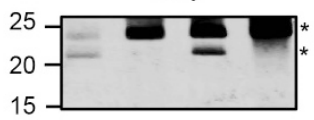

b

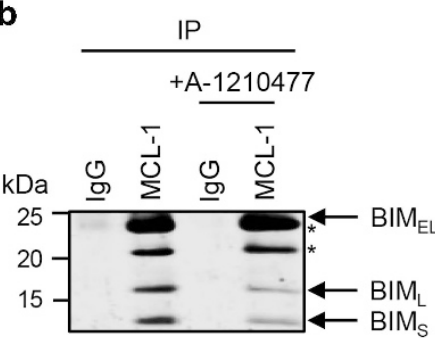

c

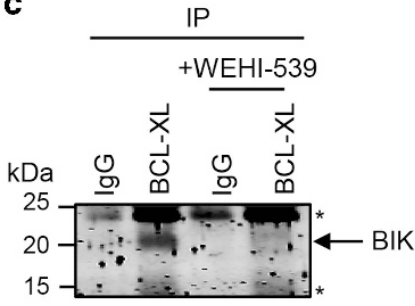

Figure 4 MCL-1 associates with BIM and BCL-XL associates with BIK in tonsil B cells. (a) Western blots after IP experiments in total B cells purified from the tonsil. Shown are the indicated proteins in the lysate after IP (cleared lysate) or the indicated proteins that were pulled down (IP). Background bands in the IP fraction were visualized by incubation with only the labeled secondary antibody and are indicated by $\left(^{*}\right)$. The experiment shown is representative of four individual experiments. (b) IP as in (a), but either with or without treatment of $30 \mu \mathrm{M} \mathrm{A}-1210477$ for $4 \mathrm{~h}$ at $37^{\circ} \mathrm{C}$. Experiment is representative of two individual experiments. (c) IP as in (a), but either with or without treatment of $10 \mu \mathrm{M}$ WEHI-539 for $4 \mathrm{~h}$ at $37^{\circ} \mathrm{C}$. Experiment is representative of two individual experiments 

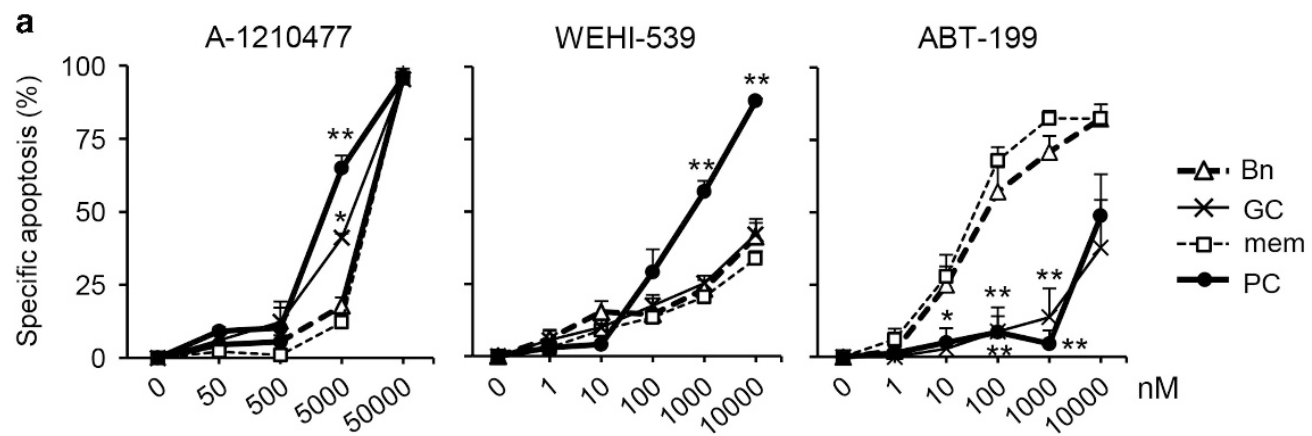

b A-1210477

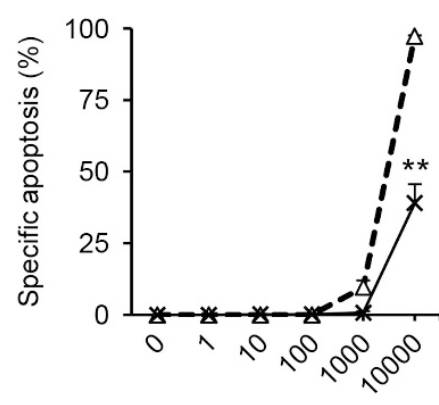

WEHI-539

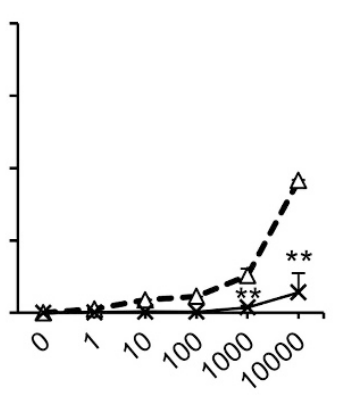

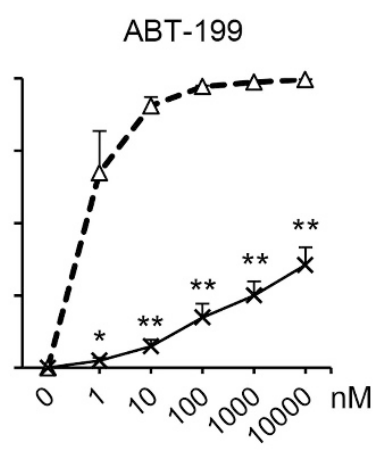

C

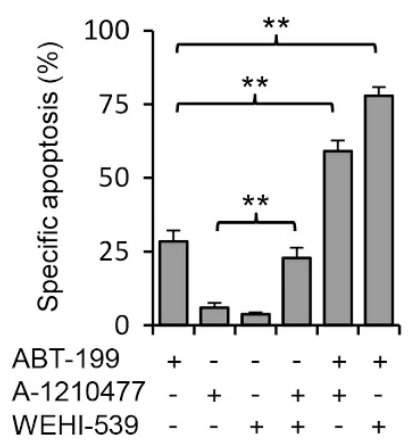

$$
-\Delta \cdot \underset{+3 \mathrm{~T} 3}{\mathrm{CLL}} \quad * \begin{gathered}
\mathrm{CLL} \\
+3 \mathrm{~T} 40
\end{gathered}
$$

Figure 5 Differential sensitivity of healthy and leukemic B cells upon inhibition of MCL-1, BCL-XL or BCL-2. (a) Total B cells purified from the tonsil were incubated with MCL-1-specific inhibitor A-1210477, BCL-XL-specific inhibitor WEHI-539 or BCL-2-specific inhibitor ABT-199 for $24 \mathrm{~h}$ at $37^{\circ} \mathrm{C}$ with indicated drug concentrations $(1 \mathrm{nM}-10 \mu \mathrm{M}$ for WEHI-539 and ABT-199, $50 \mathrm{nM}-50 \mu \mathrm{M}$ for A-1210477). B-cell populations (naive B cells; Bn, GC B cells; GC, PC or memory B cells; mem) were discriminated using antibodies recognizing $\operatorname{lgD}$ and CD38 as shown in Figure 1a. Specific apoptosis was calculated by measuring the altered percentage of TOPRO3 ${ }^{-}$(live) cells within indicated B-cell populations, compared with untreated cells. Data are average values of four different patient samples with S.E.M. and is representative of two independent experiments. Statistics were calculated in relation to Bn cells. (b) Experiment as in a, using primary CLL cells co-cultured with 3 T3 cells without (unstimulated) or with (stimulated) expression of $\mathrm{CD} 40 \mathrm{~L}(3 \mathrm{~T} 40)$ in the presence of indicated drug concentrations (1 nM-10 $\mu \mathrm{M})$. Data are average values of three different patient samples with S.E.M. and is representative of two independent experiments. Statistics were calculated in relation to unstimulated CLL cells. (c) Evaluation of combination effects of A-1210477 (5000 nM), WEHI-539 (5000 nM) or ABT-199 (1000 nM) on stimulated CLL cells. Experiment performed as in (b) with CLL cells co-cultured with $3 T 40$ cells, shown are average values of nine different patient samples with S.E.M. Statistics were calculated in relation to single treatment with indicated drug. ${ }^{*} P \leqslant 0.05,{ }^{* \star} P \leqslant 0.01$

Like GC B cells, CLL cells stimulated via CD40 have increased expression of BCL-XL, MCL-1 and BFL-1 when compared with unstimulated CLL cells that mainly express BCL-2. ${ }^{17,21}$ CLL cells can therefore be used as a model to study dependence of primary malignant $B$ cells on expression of the different BCL-2 family members, with unstimulated CLL cells behaving as naive or memory B cells and CD40stimulated CLL cells as GC B cells. However, in contrast to GC $B$ cells, CLL cells do retain their high expression of BCL-2. ${ }^{17}$ As expected based on recent studies with ABT-199, CD40stimulated CLL cells were insensitive to single treatment with specific BH3-mimetics (Figure 5b). However, different combinations of drugs were used to test potential additive or synergistic effects and revealed that ABT-199 plus WEHI-539 or ABT-199 plus A-1210477 efficiently induced apoptosis of CD40-stimulated CLL cells (Figure 5c).

\section{Discussion}

The maintenance of cancer cells is to a large extent controlled by the BCL-2-regulated or intrinsic apoptotic pathway. Targeting pro-survival BCL-2 family members using a novel class of therapeutics, called BH3-mimetics, can thus significantly improve the treatment of cancer. ${ }^{22}$ Although BH3-mimetic ABT-199 (Venetoclax) has been successfully used in clinical trials, ${ }^{20}$ there is still a lack of knowledge concerning the role and regulation of pro-survival BCL-2 proteins in healthy versus malignant $B$ cells. Our expression analyses confirm transcriptional upregulation of $B C L X$ and $B F L 1$ in GC B cells, ${ }^{12,14}$ which seems to be induced in the GC LZ and may be induced on signaling via CD40 by $T$ follicular helper cells in the $L Z$.

MCL-1 protein half-life is short in most cell types where it varies between 30 and $45 \mathrm{~min},{ }^{23-25}$ but conditions have been described for healthy ${ }^{26}$ and malignant ${ }^{27} \mathrm{~T}$ cells where proteasomal degradation of MCL-1 is inhibited, resulting in a prolonged protein half-life. Our transcriptional and protein analyses in purified cell populations demonstrate a significantly enhanced MCL-1 protein stability in the GC. As most B-cell malignancies originate in the GC, this observation is highly relevant and aberrant protein regulation of $\mathrm{MCL}-1$ may underlie the observed overexpression in FL and diffuse large B-cell lymphoma cells. ${ }^{4,28}$ The down-regulation of MCL-1specific ubiquitin ligases Mule, FBW7 and $\beta$ TrCP1, as well as the impact of PP2A-inhibitor OA on MCL-1 degradation may 
suggest their involvement in regulation of MCL-1 stability. The exact mechanism(s) of altered MCL-1 protein stability in GC B cells is subject of ongoing studies, and may include differential activity of additional kinases, phosphatases, ubiquitin ligases and deubiquitinases. ${ }^{18}$ Our finding that PP2A may be involved in the regulation of MCL-1 protein stability in GC B cells is in line with earlier findings that describe highly increased activity of phosphatases in GC B cells specifically, including PP2A. ${ }^{19}$

In addition to increased expression of BCL-XL, BFL-1 and MCL-1 in GC B cells, increased expression of pro-apoptotic BIM and BIK was also observed at both the mRNA and protein level and expression of both genes was already initiated in the $\mathrm{DZ}$ of the GC. BIK has been shown previously to be induced upon IgM ligation on $\mathrm{B}$ cells, ${ }^{29}$ and by transforming growth factor beta (TGF- $\beta$ ) in Burkitt lymphoma cell lines and GC cells. ${ }^{30}$ BIK may therefore be involved in selection of $B$ cells in the GC. More recently it was shown that not only BIK, but also BIM and NOXA are up-regulated upon B-cell receptor (BCR) stimulation and that induction of all three is important for BCRsignaling-induced cell death. ${ }^{31}$ Thus, our findings show that the induction of pro-apoptotic BIM and BIK is countered by the induction of MCL-1 and BCL-XL in those cells that, at the moment of analysis, survived the selection process in the GC.

Our IP experiments performed with purified tonsil B cells revealed preferential binding of $M C L-1$ to $B I M$ and of $B C L-X L$ to BIK in GC B cells. These findings indicate that inhibition of $M C L-1$ releases BIM that is capable of inducing apoptosis by activating $B A X$ and $B A K$, thereby explaining the central role for MCL-1 in human GC B cells. ${ }^{1}$ It also explains why inhibition of $B C L-X L$ in $G C$ B cells does not immediately result in apoptosis, as released BIK cannot directly activate BAX and BAK. ${ }^{1}$ Increased levels of free BIK may however sensitize GC $B$ cells to other apoptotic stimuli. The latter may also be the case for PC that do seem to depend on BCL-XL expression. As PC highly express BIM and PUMA, in addition to BIK, it is unclear which BH3-only protein is the direct activator of WEHI-539-induced apoptosis. An alternative option is that in PC BCL-XL does bind to apoptotic activators such as BIM, $\mathrm{BID}$ or PUMA. As the percentage of $\mathrm{PC}$ in the tonsil is very small (Supplementary Figure 5a), the contribution of binding partners for BCL-XL in PC may not have been detected in our IP experiments. Previously, we found that in contrast to GC B cells, primary CLL cells do not express BIK, but highly express NOXA. ${ }^{32}$ In CLL, MCL-1 is bound to NOXA, whereas BIM is also bound to other pro-survival proteins such as BCL-2 and $\mathrm{BCL}-\mathrm{XL}$, in addition to MCL-1. ${ }^{33}$

Thus, binding patterns of BCL-2 family members can vary between normal and malignant cell types and may cause differential sensitivity to specific BH3-mimetic drugs. The specificity of this approach was recently validated using specifically constructed cell lines, and was dubbed mitopriming. ${ }^{16}$ Our data using primary cells revealed that both GC $B$ cells and tonsillar PC are significantly more dependent on MCL-1 expression than naive and memory B cells. In contrast, only $P C$ are sensitive to inhibition of $B C L-X L$. Although longlived $\mathrm{PC}$ in the BM do not depend on BCL-XL expression, ${ }^{34}$ newly generated $P C$ and plasma blasts do. ${ }^{13,35-37}$ This supports the view that, as observed for platelets, ${ }^{38} \mathrm{BCL}-\mathrm{XL}$ acts as a survival timer for PC. BCL-XL expression induced during the $\mathrm{GC}$ reaction may protect $\mathrm{PC}$ en route to protective

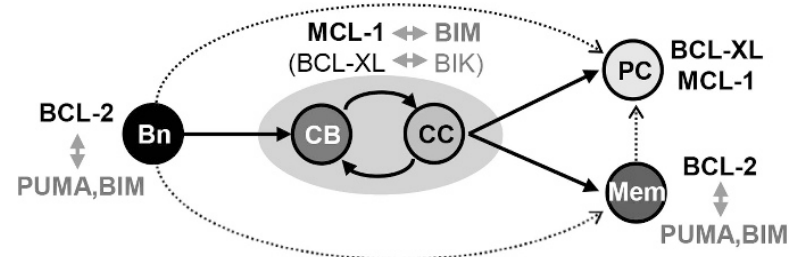

Figure 6 Model of expression and dependence on BCL-2 family proteins. Schematic representation of the dependence of naive B cells, GC B cells, PCs or memory B cells on pro-survival proteins BCL-2, BCL-XL or MCL-1 (black bold). Proapoptotic proteins that associate with indicated pro-survival proteins are show in gray. Interaction between BCL-XL and BIK in GC B cells (between brackets) seems not involved in cell survival. Arrows indicate possible routes of differentiation after activation of naive $B$ cells

BM niches where local survival signals induce expression of MCL-1. As a large portion of tonsil PC are short-lived (multiple days instead of months to years for long-lived PC), ${ }^{39}$ dependence on BCL-XL fits with this hypothesis.

$P C$ and $G C B$ cells are highly insensitive to selective inhibition of BCL-2 using ABT-199, in contrast to naive and memory $B$ cells. These findings are in line with experiments in mice where treatment with $A B T-737$ significantly reduces the naive and memory B-cell populations. ${ }^{35} \mathrm{Bfl}-1 / \mathrm{A} 1$ promotes the survival of mature follicular $B$ cells in mice, ${ }^{40,41}$ and we observed increased expression of BFL-1 in human GC B cells. The current lack of BFL-1-specific inhibitors unfortunately precludes this type of $\mathrm{BH} 3-$ mimetic profiling analysis in primary human B cells.

Combined, our experiments using healthy cells reveal a radical disparity in the expression of- and dependence onpro-survival BCL-2 family members, which is schematically summarized in Figure 6. Naive and memory B cells mainly depend on BCL-2 expression for survival. GC B cells solely depend on expression of MCL-1, whereas PC in the tonsil require expression of both $M C L-1$ and $B C L-X L$. The abundance of MCL-1, BCL-2 or BCL-XL protein correlates well with the shifts in cellular dependence. Expression of $\mathrm{MCL}-1$ in $\mathrm{GC}$ $B$ cells is important for cell survival as it sequesters BIM, whereas expression of $\mathrm{BCL}-\mathrm{XL}$ in these cells seems less important for survival as it is bound to BIK. Expression of BCL-2 in naive and memory $B$ cells promotes cell survival through sequestration of PUMA and BIM.

CLL cells directly isolated from the blood efficiently underwent apoptosis on treatment with either ABT-199, less so for WEHI-539 or A-1210477, whereas CD40-stimulated CLL cells were significantly more resistant to all three compounds. The acquired resistance can be explained by the CD40-mediated induction of BCL-XL, MCL-1, BFL-1 and overexpression of BCL-2. ${ }^{17,21}$ BCL-XL contributes significantly to ABT-199 resistance as determined by knockdown experiments in primary CLL cells. ${ }^{17}$ In line with these findings, we show here that combined use of BH3-mimetics, especially the combination of ABT-199 plus WEHI-539 or ABT-199 plus A-1210477, induced apoptosis of stimulated CLL cells much more efficiently than single drug treatment. Synergistic effects of A-1210477 and ABT-263 (navitoclax), the orally bioavailable version of BCL-2- and BCL-XL-inhibitor ABT-737, have also recently been observed for multiple cancer cell lines. ${ }^{7}$ Thus, 
increased sensitivity of primary malignant B cells to combined treatment may enlarge the therapeutic window of $\mathrm{BH} 3-$ mimetic drugs for the treatment of B-cell malignancies. These findings can be exploited, possibly using combinations of novel BH3mimetics, in therapeutic strategies targeting malignant $\mathrm{B}$ cells.

\begin{abstract}
Materials and Methods
Tonsils, CLL cells and flow cytometry. Tonsils were obtained from patients undergoing tonsillectomy for chronic tonsillitis. The tonsillar tissue was cut into small pieces and forced through a cell strainer to generate single-cell suspensions. Single-cell suspensions were stained with the following antibodies: anti-CD19 (HIB19), anti-CD3 (UCHT1), anti-IgD (IA6-2), anti-CD38 (HB7) and antiCXCR4 (12G5). Stained cells were analyzed on a FACSCanto II or LSRFortessa cytometer (Beckton Dickinson (BD) Biosciences, San Jose, CA, USA). B-cell populations were sorted using a FACSCanto II cytometer to a purity of $98 \%$. CLL cells were obtained and cryopreserved as previously described.21 The study was approved by the medical ethics committee at the Academic Medical Center and written informed consent was obtained in accordance with the Declaration of Helsinki. Expression of CD5 and CD19 (both Beckton Dickinson (BD) Biosciences) on leukemic cells was assessed by flow cytometry (FACScanto; BD Biosciences). CLL samples included in this study contained $81-99 \% \mathrm{CD}^{+} / \mathrm{CD} 19^{+}$cells. Data in Figure $5 b$ represents $C L L$ cells from three patients (two with unmutated and one with mutated $\operatorname{lgV}_{\mathrm{H}}$ gene sequences) where two patients were untreated before sampling and one patient was treated with fludarabine, cyclophosphamide and rituximab (FCR). Data in Figure $5 \mathrm{c}$ represents CLL cells from nine patients (four with unmutated and five with mutated $\operatorname{lgV}_{H}$ gene sequences) where seven patients were untreated, one patient was treated with FCR and one patient was treated with chlorambucil, cyclosporin, prednisolone and rituximab.
\end{abstract}

MLPA and qPCR. Total ribonucleic acid (RNA) for reverse transcriptase multiplex ligation-dependent probe amplification (RT-MLPA) or quantitative real time PCR was extracted using an RNA miniprep kit (Sigma, St. Louis, MO, USA). RTMLPA procedure was performed as previously described. ${ }^{2}$ qPCR was performed with Fast SYBR Green Master Mix on a StepOne Plus machine (Life Technologies, Breda, The Netherlands) using the following primers: Hprt forward $5^{\prime}$ CCTGGCGTCGTGATTAGTGA-3', Hprt reverse 5'-CGAGCAAGACGTTCAGTCCT -3', MCL1 forward 5'-TCGTAAGGACAAAACGGGAC-3', MCL1 reverse 5'-CAT TCCTGATGCCACCTTCT-3', BCL2 forward 5'-ATGTGTGTGGAGAGCGTCAA-3', BCL2 reverse 5'-CAGTTCCACAAAGGCATCCCAG-3', BCLX forward 5'GTATTGGTGAGTCGGATCGC-3', BCLX reverse 5'-TGCTGCATTGTTCCCATAGA -3', BFL1 forward 5'-TCATATTTTGTTGCGGAGTTCA-3', BFL1 reverse 5'-TCC AGCCAGATTTAGGTTCAA-3', HUWE1 forward 5'-TAATCATGCCGCAGAAGAAG -3', HUWE1 reverse 5'-CAACCTGCTGATTAGGCTCA-3', FBXW7 forward 5'ACCAACTCTCCTCCCCATTC-3', FBXW7 reverse 5'-TTTCCCAAAGAAAAAGA GCG-3', BTRC forward 5'-TGCAAGAGAAGGCACTCAAG-3', BTRC reverse $5^{\prime}$-CCTGGGTTATACAGGCATCG-3'. The results were normalized to expression of Hprt using the $\Delta \Delta \mathrm{Ct}$ method. ${ }^{34}$

Western blot. Western blotting was performed as described previously. ${ }^{17}$ Blots were probed with anti-human BCL-2 (catalog number $4223 \mathrm{~S}$, Cell Signaling, Danvers, MA, USA), BCL-XL (catalog number 2764, Cell Signaling), MCL-1 (catalog number ab32087, Abcam, Cambridge, UK), BIK (catalog number sc-10770, Santa Cruz, Santa Cruz, CA, USA), BIM (catalog number SPC-113D, StressMarq, Victoria, BC, Canada), PUMA (catalog number 4976, Cell Signaling), BID (catalog number 2002, Cell Signaling), and Actin (catalog number sc-1616, Santa Cruz). Anti-BFL-1 polyclonal rabbit antibodies were a kind gift from Prof. J Borst of the Netherlands Cancer Institute, Amsterdam, The Netherlands. IRDye 680 donkey anti-rabbit IgG or IRDye 800 donkey anti-goat IgG (Westburg, Leusden, The Netherlands) were used as secondary antibodies.

Microscopy. Paraffin embedded tissue sections obtained from the Academic medical center pathology department were de-waxed by immersion in xylene and hydrated by serial immersion in ethanol and phosphate-buffered solution (PBS). Antigen retrieval was performed by heating for $20 \mathrm{~min}$ in sodium citrate buffer (10 mM sodium citrate, $0.05 \%$ Tween20, pH 6). Sections were washed with PBS and blocked (Tris-buffered saline containing 10\% bovine serum albumin and $0.3 \%$ Triton X-100) for $1 \mathrm{~h}$, and incubated with primary antibody: anti-CD20 (catalog number 14-0202, eBioscience, San Diego, CA, USA) and anti-MCL-1, in SignalStain
(Cell Signaling) overnight at $4^{\circ} \mathrm{C}$. Slides were washed with PBS and incubated with Alexa Fluor 488 labeled goat anti-mouse, Alexa Fluor 594 labeled goat anti-rabbit antibodies (1/400, Invitrogen, Carlsbad, CA, USA) for $1 \mathrm{~h}$, and stained for $10 \mathrm{~min}$ with $4^{\prime}, 6$-Diamidino-2-phenylindole $(0.1 \mu \mathrm{g} / \mathrm{ml}$ in PBS). Sections were mounted with Fluoromount-G (eBioscience) and immunofluorescence imaging was performed using a Leica DMRA fluorescence microscope equipped with a cooled camera. Images were acquired using Image Pro Plus (Media Cybernetics, Rockville, MD, USA) and composed in Adobe Photoshop CS3.

B-cell enrichment and IPs. B cells from the tonsil were purified using a Dynabeads Untouched human B cells kit (catalog number 11351D, ThermoFisher, Waltham, MA, USA) and subjected to IP using a Dynabeads protein G immunoprecipitation kit (catalog number 10007D, ThermoFisher) and antibodies described in the western blot section.

BH3-mimetics and apoptosis assays. Purified B cells from the tonsil were cultured with indicated concentrations of A-1210477 (catalog number CT-A121, ChemieTek, Indianapolis, IN, USA), WEHI-539 (catalog number 1431866-33-9, MedChem Express, Monmouth Junction, NJ, USA) or ABT-199 (catalog number A0776, LKT Laboratories, St Paul, MN, USA) for $24 \mathrm{~h}$ at $37^{\circ} \mathrm{C}$. Cell viability after treatment was assessed by flow cytometry using the TO-PRO-3 dye (catalog number T3605, ThermoFisher). Specific apoptosis was calculated by measuring the altered percentage of TOPRO3 ${ }^{-}$(live) cells within indicated B-cell populations, compared with untreated cells and is defined as (\% cell death in treated cells $)-(\%$ cell death in medium control) / (\% viable cells medium control) $\times 100$.

Statistical analysis. Statistical significance was determined using a two-tailed Student's $t$-test. $P$-values $<0.05$ were considered statistically significant.

\section{Conflict of Interest}

The authors declare no conflict of interest.

Acknowledgements. We thank the facilities of our institute, and particularly B Hooibrink and T van Capel for flow cytometric cell sorting, the department of Otorhinolaryngology for donation of tonsils as leftover material after tonsillectomies and FM Wensveen and MHJ van Oers for critical reading of the manuscript. We are grateful to D Luijks for technical assistance with the MLPA experiments and to JP Medema and CD Zimberlin for the use of WEHI-539. This work was supported by a ZonMw-VENI grant (Number 91614046) to VP.

1. Czabotar PE, Lessene G, Strasser A, Adams JM. Control of apoptosis by the BCL-2 protein family: implications for physiology and therapy. Nat Rev Mol Cell Biol 2014; 15: 49-63.

2. Peperzak V, Vikstrom IB, Tarlinton DM. Through a glass less darkly: apoptosis and the germinal center response to antigen. Immunol Rev 2012; 247: 93-106.

3. Souers AJ, Leverson JD, Boghaert ER, Ackler SL, Catron ND, Chen J et al. ABT-199, a potent and selective BCL-2 inhibitor, achieves antitumor activity while sparing platelets. Nat Med 2013; 19: 202-208.

4. Schwickart M, Huang X, Lill JR, Liu J, Ferrando R, French DM et al. Deubiquitinase USPgX stabilizes MCL1 and promotes tumour cell survival. Nature 2010; 463: 103-107.

5. Kitada S, Andersen J, Akar S, Zapata JM, Takayama S, Krajewski S et al. Expression of apoptosis-regulating proteins in chronic lymphocytic leukemia: correlations with in vitro and in vivo chemoresponses. Blood 1998; 91: 3379-3389.

6. Kilpinen S, Autio R, Ojala K, lljin K, Bucher E, Sara H et al. Systematic bioinformatic analysis of expression levels of 17,330 human genes across 9,783 samples from 175 types of healthy and pathological tissues. Genome Biol 2008; 9: R139.

7. Leverson JD, Zhang H, Chen J, Tahir SK, Phillips DC, Xue J et al. Potent and selective smallmolecule MCL-1 inhibitors demonstrate on-target cancer cell killing activity as single agents and in combination with ABT-263 (navitoclax). Cell Death Dis 2015; 6: e1590.

8. Lessene G, Czabotar PE, Sleebs BE, Zobel K, Lowes KN, Adams JM et al. Structure-guided design of a selective BCL-X(L) inhibitor. Nat Chem Biol 2013; 9: 390-397.

9. Küppers R. Mechanisms of B-cell lymphoma pathogenesis. Nat Rev Cancer 2005; 5: $251-262$.

10. Krajewski S, Krajewska M, Shabaik A, Wang HG, Irie S, Fong L et al. Immunohistochemical analysis of in vivo patterns of Bcl-X expression. Cancer Res 1994; 54: 5501-5507.

11. Krajewski S, Bodrug S, Gascoyne R, Berean K, Krajewska M, Reed JC. Immunohistochemical analysis of Mcl-1 and Bcl-2 proteins in normal and neoplastic lymph nodes. Am J Pathol 1994; 145: 515-525. 
12. Tuscano JM, Druey KM, Riva A, Pena J, Thompson CB, Kehrl JH. Bcl-x rather than Bcl-2 mediates CD40-dependent centrocyte survival in the germinal center. Blood 1996; 88: 1359-1364.

13. Vikstrom I, Carotta S, Luthje K, Peperzak V, Jost PJ, Glaser S et al. Mcl-1 is essential for germinal center formation and B cell memory. Science 2010; 330: 1095-1099.

14. Victora GD, Dominguez-Sola D, Holmes AB, Deroubaix S, Dalla-Favera R, Nussenzweig MC. Identification of human germinal center light and dark zone cells and their relationship to human B-cell lymphomas. Blood 2012; 120: 2240-2248.

15. Del Gaizo Moore V, Letai A. BH3 profiling-measuring integrated function of the mitochondrial apoptotic pathway to predict cell fate decisions. Cancer Lett 2013; 332: 202-205.

16. Lopez J, Bessou M, Riley JS, Giampazolias E, Todt F, Rocheqüe T et al. Mito-priming as a method to engineer Bcl-2 addiction. Nat Commun 2016; 7: 10538.

17. Thijssen $\mathrm{R}$, Slinger $\mathrm{E}$, Weller $\mathrm{K}$, Geest $\mathrm{CR}$, Beaumont $\mathrm{T}$, van Oers $\mathrm{MH}$ et al. Resistance to ABT-199 induced by microenvironmental signals in chronic lymphocytic leukemia can be counteracted by CD20 antibodies or kinase inhibitors. Hematologica 2015; 100: e302-e306.

18. Mojsa B, Lasso I, Desagher S. Mcl-1 ubiquitination: unique regulation of an essential survival protein. Cells 2014; 3: 418-437.

19. Khalil AM, Cambier JC, Shlomchik MJ. B cell receptor signal transduction in the GC is shortcircuited by high phosphatase activity. Science 2012; 336: 1178-1181.

20. Roberts AW, Davids MS, Pagel JM, Kahl BS, Puwada SD, Gerecitano JF et al. Targeting BCL2 with venetoclax in relapsed chronic lymphocytic leukemia. N Engl J Med 2016; 374: 311-322.

21. Hallaert DY, Jaspers A, van Noesel CJ, van Oers MH, Kater AP, Eldering E. c-Abl kinase inhibitors overcome CD40-mediated drug resistance in CLL: implications for therapeutic targeting of chemoresistant niches. Blood 2008; 112: 5141-5149.

22. Delbridge ARD, Strasser A. The BCL-2 protein family, BH3-mimetics and cancer therapy. Cell Death Differ 2015; 22: 1071-1080.

23. Zhong Q, Gao W, Du F, Wang X. Mule/ARF-BP1, a BH3 only E3 ubiquitin ligase, catalyses the polyubiquitination of $\mathrm{Mcl}-1$ and regulates apoptosis. Cell 2005; 121 1085-1095.

24. Okamoto T, Coultas L, Metcalf D, van Delft MF, Glaser SP, Takiguchi M et al. Enhanced stability of Mcl1, a prosurvival Bcl2 relative, blunts stress-induced apoptosis, causes male sterility, and promotes tumorigenesis. Proc Natl Acad Sci U S A 2014; 111: 261-266.

25. Nijhawan D, Fang M, Traer E, Zhong Q, Gao W, Du F et al. Elimination of Mcl-1 is required for the initiation of apoptosis following ultraviolet irradiation. Genes Dev 2003; 17: 1475-1486.

26. Wensveen FM, Alves NL, Derks IA, Reedquist KA, Eldering E. Apoptosis induced by overall metabolic stress converges on the Bcl-2 family proteins Noxa and Mcl-1. Apoptosis 2011; 16 : 708-721.

27. Inuzuka H, Shaik S, Onoyama I, Gao D, Tseng A, Maser RS et al. SCF(FBW7) regulates cellular apoptosis by targeting MCL1 for ubiquitination and destruction. Nature 2011; 471: 104-109.

28. Wertz IE, Kusam S, Lam C, Okamoto T, Sandoval W, Anderson DJ et al. Sensitivity to antitubulin chemotherapeutics is regulated by MCL1 and FBW7. Nature 2011; 471:110-114.

29. Jiang A, Clark EA. Involvement of Bik, a proapoptotic member of the Bcl-2 family, in surface IgM-mediated B cell apoptosis. J Immunol 2001; 166: 6025-6033.

30. Spender LC, O'Brien DI, Simpson D, Dutt D, Gregory CD, Allday MJ et al. TGF-beta induces apoptosis in human B cells by transcriptional regulation of BIK and BCL-XL. Cell Death Differ 2009; 16: 593-602.
31. Carter MJ, Cox KL, Blakemore SJ, Bogdanov YD, Happo L, Scott CL et al. BCR-signalinginduced cell death demonstrates dependency on multiple $\mathrm{BH} 3$-only proteins in a murine model of B-cell lymphoma. Cell Death Differ 2016; 23: 303-312.

32. Mackus WJM, Kater AP, Grummels A, Evers LM, Hooijbrink B, Kramer MH et al. Chronic lymphocytic leukemia cells display p53-dependent drug-induced Puma upregulation. Leukemia 2005; 19: 427-434.

33. Hallaert DY, Spijker R, Jak M, Derks IA, Alves NL, Wensveen FM et al. Crosstalk among Bcl-2 famliy members in B-CLL: seliciclib acts via the Mcl-1/Noxa axis and gradual exhaustion of Bcl-2 protection. Cell Death Differ 2007; 14: 1958-1967.

34. Belnoue E, Pihlgren M, McGaha TL, Tougne C, Rochat AF, Bossen C et al. APRIL is critical for plasmablast survival in the bone marrow and poorly expressed by early-life bone marrow stromal cells. Blood 2008; 111: 2755-2764.

35. Carrington EM, Vikstrom IB, Light A, Sutherland RM, Londrigan SL, Mason KD et al. BH3 mimetics antagonizing restricted prosurvival $\mathrm{Bcl}-2$ proteins represent another class of selective immune modulatory drugs. Proc Natl Acad Sci U S A 2010; 107: 10967-10971.

36. Peperzak V, Vikstrom I, Walker J, Glaser SP, LePage M, Coquery CM et al. Mcl-1 is essential for the survival of plasma cells. Nat Immunol 2013; 14: 290-297.

37. Gaudette BT, Iwakoshi NN, Boise LH. Bcl-xL protein protects from C/EBP homologous protein (CHOP)-dependent apoptosis during plasma cell differentiation. J Biol Chem 2014; 289: 23629-23640.

38. Mason KD, Carpinelli MR, Fletcher Jl, Collinge JE, Hilton AA, Ellis S et al. Programmed anuclear cell death delimits platelet life span. Cell 2007; 128: 1173-1186.

39. van Laar JM, Melchers M, Teng YK, van der Zouwen B, Mohammadi R, Fischer R et al. Sustained secretion of immunoglobulin by long-lived human tonsil plasma cells. Am J Pathol 2007; 171: 917-927.

40. Ottina E, Grespi F, Tischner D, Soratroi C, Geley S, Ploner A et al. Targeting antiapoptotic A1/Bfl-1 by in vivo RNAi reveals multiple roles in leukocyte development in mice. Blood 2012; 119: 6032-6042.

41. Sochalska M, Ottina E, Tuzlak S, Herzog S, Herold M, Villunger A. Conditional knockdown of BCL2A1 reveals rate-limiting roles in BCR-dependent B-cell survival. Cell Death Differ 2016; 23: 628-639.

42. Eldering E, Spek CA, Aberson HL, Grummels A, Derks IA, de Vos AF et al. Expression profiling via novel multiplex assay allows rapid assessment of gene regulation in defined signalling pathways. Nucleic Acids Res 2003; 31: e153.

This work is licensed under a Creative Commons Attribution-NonCommercial-NoDerivs 4.0 International License. The images or other third party material in this article are included in the article's Creative Commons license, unless indicated otherwise in the credit line; if the material is not included under the Creative Commons license, users will need to obtain permission from the license holder to reproduce the material. To view a copy of this license, visit http://creativecommons.org/licenses/by-nc-nd/4.0/

(C) The Author(s) 2017

Supplementary Information accompanies this paper on Cell Death and Differentiation website (http://www.nature.com/cdd) 